\title{
Design and development of the home healthcare worker observation tool
}

\author{
Elizabeth Ann Bien*1, Kermit G. Davis ${ }^{2}$, Tamara F. Small ${ }^{1}$, Susan Reutman ${ }^{2}$, Gordon L. Gillespie ${ }^{1}$ \\ ${ }^{1}$ College of Nursing, University of Cincinnati, United States \\ ${ }^{2}$ Department of Environmental and Public Health Sciences, University of Cincinnati, United States
}

Received: March 15, 2021

DOI: $10.5430 /$ jnep.v11n9p29
Accepted: April 27, 2021

URL: https://doi.org/10.5430/jnep.v11n9p29

\begin{abstract}
Background: The rapidly growing number of home healthcare workers (HHCW) are exposed to unique occupational hazards within each patient home. This article describes the development of an observation tool to document occupational hazards HHCWs encounter.

Methods: Tool development followed three steps: determining content domain, content validity, and inter-rater agreement. Results: Expert feedback guided the revision of content domain to 636 items. Scale level content validity index (S-CVI) was 0.90 . Inter-rater agreement tests resulted in percent agreement and accuracy mean of $89.5 \%$ and frequency variables resulted in standard deviations from 0 to 8.62 .

Conclusions: The observation tool encompasses the diverse range of occupational hazards HHCWs encounter; inter-rater percent agreement and overall accuracy scores were acceptable. Future pilot testing of this tool among broader raters and populations is recommended to characterize its usability, internal consistency, and reliability to assess HHCW occupational hazards.
\end{abstract}

Key Words: Workplace, Home health, Home care, Occupational health, Content validity, Environmental exposures, Ergonomic exposures

\section{INTRODUCTION}

The home healthcare industry is growing rapidly, resulting in expansion of the number of workers employed. Due to an aging population with chronic conditions, more persons are seeking care. More people are wanting to be at home during chronic illnesses and new technologies allow for higher levels of care to be provided in the home. The U.S. Bureau of Labor Statistics ${ }^{[1]}$ reports a $29.9 \%$ increase in number of home health care service industry employees from 2019 to 2029. The type of workers providing care to persons within their home range from personal care aides, who provide assistance with activities of daily living such as cooking, cleaning, dressing, or bathing within the patient home, to licensed healthcare professionals such as nurses, respiratory therapists, physical therapists, occupational therapists, social workers, and medical providers. Within this document all types of workers going into the home to provide care are referenced under the term home healthcare workers (HHCWs). HHCWs encounter occupational hazards based on the type of care they provide and the unique hazards within each home, leading to occupational injuries and illnesses. ${ }^{[2]}$

\section{BACKGROUND}

2.1 Occupational injuries and illnesses of home healthcare workers

HHCWs have increased rates of asthma, with a prevalence rate of $13.2 \%$, compared to the prevalence of $7.2 \%$ for all U.S. workers combined according to National Institute for

*Correspondence: Elizabeth Ann Bien; Email: bienea@ucmail.uc.edu; Address: College of Nursing, University of Cincinnati, United States. 
Occupational Safety and Health (NIOSH). ${ }^{[3]}$ In addition to asthma, the BLS ${ }^{[4]}$ reports the incidence of non-fatal occupational injuries and illnesses was 2.7 per 100 full time HHCWs, which is considerably lower than the incidence rate for hospital workers at 5.6 per 100 full time workers. It is surprising that HHCWs report lower injury and illness incidence rates than hospital workers given that they often work within a large variety of environments such as inside different homes and outside to and from their vehicle. ${ }^{[2]}$ These concerns have been noted within other studies. Davis et al. ${ }^{[5]}$ indicated previous studies focus on low back pain of hospital workers, with musculoskeletal research lacking evidence related to musculoskeletal disorder of home healthcare workers and injuries of the extremities. Several researchers indicated needlestick exposures are underreported. Gershon et al. ${ }^{[6]}$ found that home healthcare registered nurses underreported needlesticks at a rate of $35 \%$. Quinn et al. ${ }^{[7]}$ estimate underreporting to be closer to $50 \%$.

\subsection{Findings and limitations of previous research}

Previous studies have been completed to assess the occupational hazards HHCWs encounter and procedures include surveys, interviews, and focus groups, which used tools or surveys that were validated and piloted.$^{[8-11]}$ Occupational hazards that align within many studies include: exposure to bloodborne pathogens; slip, trip, and fall hazards walking to and within the home; second hand smoke; respiratory exposures including cleaning chemicals, pets, and pests; aggression from pets and persons; and ergonomic hazards providing care within the unplanned environment with limited access to patient care devices such as lifts. ${ }^{[2,8-10,12-14]}$ While there is agreement on the types of hazards workers report encountering in the home care environment, there are limitations to the studies.

Many of the previous study designs were limited to selfreport, potentiating recall bias and uncertainty of validity and accuracy due to workers' perceptions of hazards. ${ }^{[2]}$ The Centers for Disease Control and Prevention (CDC $)^{[15]}$ indicates a benefit to having workers report site hazards and emphasizes the necessity of formal worksite environmental assessments. While self-report is often an easier method to gather information about hazards, reported perceptions are potentially biased. A better understanding of actual hazards requires first-hand knowledge of the occupational environment of home care to fully understand the conditions of work. ${ }^{[15]}$ Direct observation by researchers are one way to gather this first-hand knowledge and the development of an observation tool is a logical next step.

\subsection{Conceptual model supporting the foundation for an observation tool}

It was important to design a study that documents the variables within the workplace that have the potential to impact the health outcomes of HHCWs and identify a conceptual model that informs this research. Design of the study is based on a conceptual model for Integrated Approach for Worker Health and Safety was developed by Harvard T.H. Chan School of Public Health. ${ }^{[16]}$ This conceptual framework was developed to define and guide research, policies, and practices that influence occupational health and safety. The physical environment, organization of work, psychological factors, and job tasks and demands, are referred to as the "conditions of work" and have an impact on worker outcomes such as illness, injury, and wellbeing. ${ }^{[16]}$ The design of the current study was based on this conceptual framework, specifically identifying the conditions of work within each patient home that have the potential to individually or cumulatively impact worker outcomes.

Establishing a better understanding of the occupational hazards and conditions of work that the growing industry of HHCWs encounter requires observing the workers in their work environment. To understand the workplace exposures, the Home Healthcare Worker Observation Tool was developed. The design of the tool followed three steps: determining content domain, content validity, and inter-rater agreement. The purpose of this paper was to describe the tool development stages of the observation tool that will be used to document occupational hazards specific to HHCWs. Once developed, the use of the tool will be piloted with a researcher following a $\mathrm{HHCW}$ during patient visits and using the tool as a checklist to assess the physical environment and job tasks of HHCWs.

\section{Methods}

Content development, content validity, and interrater agreement of the Home Healthcare Worker Observation tool were the initial steps of a study aimed to describe the occupational exposures HHCWs encounter within the home care environment. This project was approved by the Institutional Review Board prior to implementation.

\subsection{Stage 1: Content development for instrument}

The tool development team included an advanced practice nurse with over 10 years of experience in home healthcare and occupational health; a research expert with a focus on mental and physical workplace demands and health outcomes, specifically ergonomic stressors in healthcare; a nurse scientist with a homecare background and research focus on workplace violence in healthcare; and a nurse with clinical 
experience in infection control in the acute care setting and occupational health nursing.

According to Beck and Gable, ${ }^{[17]}$ an initial step of scale development is establishing content domain and defining attributes that are intended to be measured by the scale. Lynn ${ }^{[18]}$ further identified a literature review was needed to determine dimensions and sub- dimensions relevant to the content domain; expressed that a literature review not be the sole basis for the items and that clinicians working in the field could provide valuable insight. For this study, a literature review was completed following the guidelines of Whittemore and Knaff ${ }^{[19]}$ to identify the breadth of occupational hazards HHCWs encounter. Experts then were consulted and interviewed about their current knowledge and experience working within home healthcare. The experts consisted of those with research or clinical knowledge of home care as an occupational setting including several clinicians, a researcher, a home healthcare accreditation specialist, and an occupational health and safety expert.

Following a thorough literature review, an a priori procedure for item development was applied to identify essential sections and variables specific to establishing the content domain of occupational hazards in the home care environment. The literature review led to the development of 12 sections including safety and safety training; characteristics of the patient and patient home; slip, trip, and fall hazards; sharps and bloodborne pathogens; environmental exposures; hand hygiene; ingress and egress with equipment; community surveillance and violence; injuries and incidents; ergonomic hazards associated with specific tasks; hazards associated with medication administration and procedures; and chemical exposures.

It was determined by the research team that the items within the tool needed to include hazards beginning when the HHCW exited their vehicles, assessing items that may be a hazard within the community, walking into and working within the patient home, and ending with the worker getting back into the vehicle. Hazards that occur within the vehicles or related to travel for this work group is outside the scope of this tool. After the observation tool was developed, expert content validity indexing was sought for a posteriori judgement of the items.

\subsection{Stage 2: Content validity}

Once the items in the scale were developed, it was necessary to complete content validity assessment to ascertain if items appropriately represented the content domain, adding or removing items as needed. ${ }^{[17]}$ For this step, a total of 6 experts were engaged: three experts with research related to occu-

Published by Sciedu Press pational hazards within the home healthcare environment, two occupational health nurses with previous experience in clinical care in the home, and one occupational health nurse and employee health manager within a home care agency. The experts were invited to review and rate each item of the observation tool, assessing the relevance of the occupational hazards in the home care environment to determine if the items placed on the observation tool adequately reflected the breadth of potential occupational exposures of HHCWs.

Assessing the content validity of the tool followed the process described by Lynn. ${ }^{[18]}$ A four-point rating scale was used, (1) indicating not relevant to (4) indicating very relevant. The index of content validity (CVI) allowed the researcher to determine if an item is relevant or not relevant to assessing occupational hazards in the home care environment. An item level CVI was determined by dividing those items with a rating of 3 or 4 , by the number of experts providing a rating. ${ }^{[18]}$ Items with a CVI of 0.83 or higher was considered acceptable, and an overall scale CVI (S-CVI) of 0.80 or higher was acceptable. ${ }^{[19,20]}$ A comments section on the rating scale allowed the expert to provide qualitative feedback and make suggestions of any item that needed to be added or clarified to fully describe the hazards HHCWs encounter. These comments allowed the team to identify omissions and needed refinement of the items within the instrument. Table 1 displays the 12 sections, corresponding CVI, and number of items within each section before and after CVI.

\subsection{Stage 3: Inter-rater agreement}

As multiple raters were to be engaged in this study and the number of observers increases the potential for measurement error, it was important to assess the level of agreement between the raters prior to data collection. ${ }^{[21,22]}$ Inter-rater agreement allowed the researchers to assess the consistency of the raters using the tool and identify areas of variability. Within this study, the four raters were graduate students enrolled in an interdisciplinary workshop course focused on occupational health and safety research, specifically focusing on research of HHCWs and occupational hazards. Inter-rater agreement was used to ensure the instrument is measuring the presence of an occupational hazard and not the difference in rater's perception of the presence of a hazard. In this study, simulated patients and home care environments were used for training and assessment of inter-rater agreement.

\subsubsection{Rater training}

Simulated patients and scenarios were used to depict real patient situations. ${ }^{[23]}$ To instruct and demonstrate the application and use of the tool in a home care environment, a 30-minute narrated training tool was developed with the use of photos mimicking the home healthcare environment and 
descriptions of items within the tool. One example of this is a description and photo of an "improvised sharps container", which is an item on the sharps section of the tool. A photo of an improvised sharps container made for the training tool is shown in Figure 1. At the conclusion of the 30-minute train- ing, two videos illustrating patient care were linked and raters were encouraged to watch the videos and practice using the observation tool as a checklist to document the environmental hazards and tasks they observed. This simulates how the researcher will use the tool in the home care environment.

Table 1. Items Within Each Section Before and After Content Validity

\begin{tabular}{llll}
\hline & & Number of Items & Number of Items \\
\cline { 2 - 4 } & CVI & Before CVI & After CVI \\
\hline Safety \& Safety Training & .67 & 12 & 8 \\
Type of Patient \& Home & .83 & 24 & 17 \\
Slip/Trip/Fall & 1 & 17 & 19 \\
Sharps & 1 & 7 & 7 \\
Environmental & .83 & 24 & 28 \\
Hand Hygiene & 1 & 6 & 8 \\
Ingress/Egress & .83 & 1 & 1 \\
Community Surveillance/Threats/Abuse & .83 & 41 & 39 \\
*Injury/Incident & 1 & 12 & 5 \\
*Ergonomics & .83 & 154 & 145 \\
*Medications/Procedures & 1 & 160 & 160 \\
*Cleaning/Chemical Use & 1 & 199 & 199 \\
Total CVI for Relevancy of & .90 & & \\
All Items Within the Scale & & & 636 \\
Total \# of Items & & 657 & \\
\hline
\end{tabular}

Note. The sections marked with an asterisk (*) are only used when workers perform certain tasks.

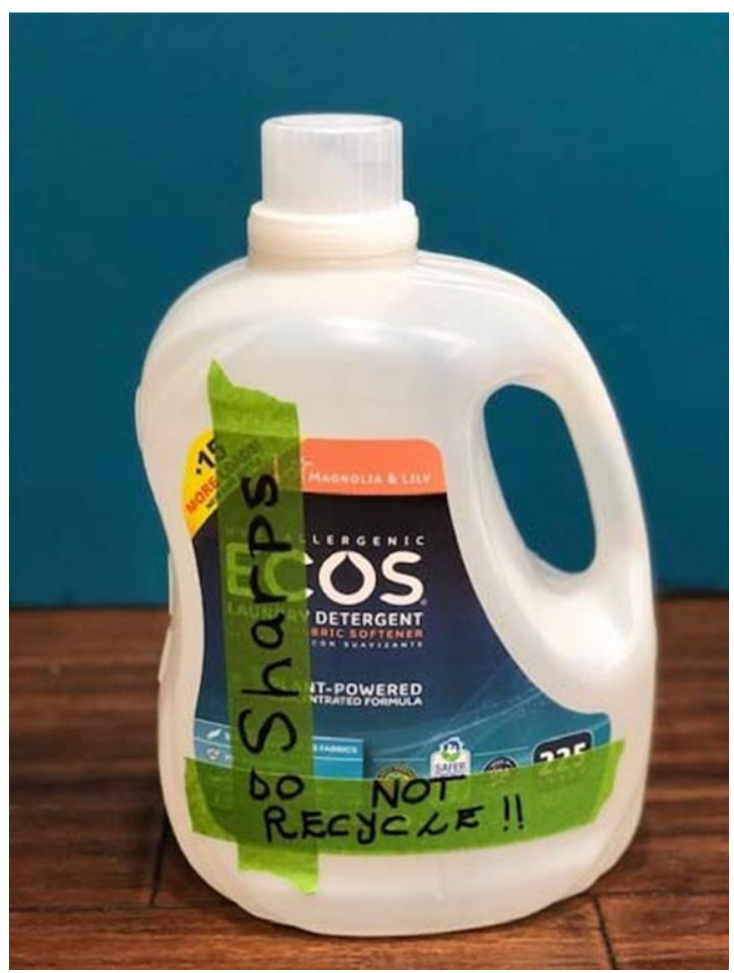

Figure 1. Improvised Sharps Container

\subsubsection{Assessment}

In collaboration with a residential retirement community and the simulation lab staff from a nursing school, items were obtained to develop realistic scenarios to simulate the patient care environment in a home care setting. For example, one video simulated a HHCW assisting a patient with a urinary catheter from a bed to a wheelchair within a very narrow working space. In another video, the worker moved a chair and straightened a throw rug prior to sitting with the patient to assist with a medication injection. Video recordings of the scenarios provided consistency of the simulations. The online format of training and inter-rater agreement assessment allowed raters to complete assessment at their own pace, when and where their schedule allocated time, and independent of other raters.

Raters were given the assessment sheet, including instructions on how to document the assessment, and access the video links. The assessment sheet was printed to allow raters to use the paper and pen method they will use in the field. Raters provided checkmarks for observed hazards (e.g. "cluttered walkway") and hashmarks where appropriate for items that required documentation of frequency (e.g. number of 
times HHCW was observed to "reach" during patient care).

Inter-rater agreement (IRA) using percent agreement is used for nominal or categorical variables to determine the level of agreement between multiple raters of the value being observed. ${ }^{[21]}$ For this study, IRA across the raters were compared for categorical variables that were either observed or not observed. A score was given across the four raters as a percentage, indicating percent of agreement amongst the raters. For example, a laundry basket is present in a walkway and the raters were to indicate if clutter was observed. If all four raters indicate clutter is observed, the percent agreement is $100 \%$. If three of the four raters indicate clutter is observed, the percent agreement is $75 \%$.

Gisev et al. ${ }^{[21]}$ indicates that IRA can be interpreted in a number of ways and that a predetermined level of agreement is not appropriate for all studies. Gisev et al. ${ }^{[21]}$ cautions that IRA does not indicate if the raters are correct in their answers. Therefore, an accuracy percent was also calculated for categorical items. For example, if the walkway was clear and two of the four raters incorrectly indicated there was clutter in the walkway, the accuracy would be $50 \%$. For this study, it was determined that after completion of the assessment, the assessment results would be reviewed with the group of raters, paying close attention to review items with less than $100 \%$ agreement and accuracy, ensuring raters understand the occupational hazards and appropriate documentation on the observation tool.

Included within the tool were items that required the use of documenting the observed frequency of certain ergonomic positions that can potentiate musculoskeletal injuries. An example of this includes the number of times a worker is observed "kneeling" when completing an assessment. For items documented by each observer as a frequency, analysis across the raters included mean and standard deviation.

\section{Results}

\subsection{Stage 1: Content development for instrument}

The literature review and consultation of persons with practical and expert knowledge of the home care environment provided a range of potential occupational hazards to be included in the observation tool. Due to the robust number of individual items within the tool, items were divided into 12 sections. The sections included safety and safety training; type of home and type of patient; slip, trip, and fall hazards; sharps; environmental; hand hygiene; ingress and egress with equipment; community surveillance, threats, and abuse; injury and incidents; ergonomics; medications and procedures; and cleaning and chemical use. Within these sections there were 657 items. The ability of these items to represent the content domain of occupational hazards in the home care environment was further determined using the CVI.

\subsection{Stage 2: Content validity}

For the 12 sections within the tool, CVI ranged from 0.67 to 1.0 , with most items falling within the acceptable limit of 0.83 or higher as determined by Lynn. ${ }^{[18]}$ The safety and safety training section was 0.67 , falling below the acceptable limit, and after input from expert reviewers and discussion with the research team, 1 item was moved to the demographic sheet and 3 items were removed as they required inquiry and were not strictly observable. The overall S-CVI is 0.90 , above the acceptable limit for an overall scale CVI (S-CVI) of 0.80 or higher. ${ }^{[18,20]}$ Table 1 shows the CVI for each section and an overall S-CVI and a summary of the number of items within each section before and after assessment of content validity. Within the sections, 21 items were removed based on expert feedback.

As described by Lynn, ${ }^{[18]}$ any items that are absent from the tool must be identified and those omissions should be addressed through further clarification. An area for comments on the tool allowed for such feedback of omissions during the assessment of content validity. Through identification of these omissions, 14 items were added to the tool within the sections.

Items that were added or clarified include addition of "stairs without railings", "water or grease on the floor", "used needles recapped", "dried hands with reusable towel", "dried hands with disposable towel", other types of odors in the home, and an area for charting any type of precipitation. "Other" was added to the section related to tobacco smoke, and "drug paraphernalia" was added to capture "other" types of drug use occurring in the home. A prompt to "call 911" when certain types of violence were observed was added. Within the ergonomics section, items were added to capture ergonomic risks during patient assessment and care.

It was important that items retained on the observation tool were observable as the premise of the project is to observe the worker in the patient care environment, having limited interaction with the patient and worker during the observations. Items removed due to lack of clarity or that were considered ambiguous by the experts were "vehicle well maintained"; "GPS navigation used"; and "clear driving directions". Items removed due to irrelevance and no access to the patients' medical chart included if the patient was being seen for certain types of illness such as "acute", "chronic", and "mental health". Also removed was "type of insurance". The final Home Healthcare Observation Tool can be found in Appendix A. 
Table 2. Percent Agreement and Accuracy by Variable

\begin{tabular}{|c|c|c|}
\hline Variable Identified Within Each Video & \% Agreement & $\%$ Accuracy \\
\hline \multicolumn{3}{|l|}{ Video A } \\
\hline Dwelling & $100 \%$ & $100 \%$ \\
\hline Neighborhood & $75 \%$ & $75 \%$ \\
\hline Cluttered Walkway Outside & $75 \%$ & $75 \%$ \\
\hline Stairs without Railing & $75 \%$ & $75 \%$ \\
\hline Cluttered Walkway Inside & $75 \%$ & $75 \%$ \\
\hline Throw Rug & $75 \%$ & $75 \%$ \\
\hline \multicolumn{3}{|l|}{ Video B } \\
\hline Throw Rug & $100 \%$ & $100 \%$ \\
\hline \multicolumn{3}{|l|}{ Video C } \\
\hline Hand Sanitizer After Patient Care & $100 \%$ & $100 \%$ \\
\hline Throw Rug Inside & $100 \%$ & $100 \%$ \\
\hline Non-Safety Needle & $75 \%$ & $75 \%$ \\
\hline Sharps Container Used & $100 \%$ & $100 \%$ \\
\hline Used Needle Re-capped & $100 \%$ & $100 \%$ \\
\hline \multicolumn{3}{|l|}{ Video D } \\
\hline Gloves changing linens & $100 \%$ & $100 \%$ \\
\hline Gloves catheter care & $100 \%$ & $100 \%$ \\
\hline Badge Present & $75 \%$ & $75 \%$ \\
\hline Last name on badge & $75 \%$ & $75 \%$ \\
\hline Working alone & $75 \%$ & $75 \%$ \\
\hline Object around the neck & $100 \%$ & $100 \%$ \\
\hline \multicolumn{3}{|l|}{ Video $\mathbf{F}$} \\
\hline Manufacture Container & $50 \%$ & $50 \%$ \\
\hline Wipe disinfectant & $100 \%$ & $100 \%$ \\
\hline Furniture cleaned & $100 \%$ & $100 \%$ \\
\hline Gloves & $100 \%$ & $100 \%$ \\
\hline Active smoking & $100 \%$ & $100 \%$ \\
\hline Safety Needle Used & $100 \%$ & $100 \%$ \\
\hline Improvised Sharps Container Used & $100 \%$ & $100 \%$ \\
\hline Hands washed with soap and water before contact with patient & $75 \%$ & $75 \%$ \\
\hline Hands dried with disposable towel before contact with patient & $75 \%$ & $75 \%$ \\
\hline Electrical cord trip hazard & $100 \%$ & $100 \%$ \\
\hline Throw Rug Inside & $100 \%$ & $100 \%$ \\
\hline Gloves worn during injection & $100 \%$ & $100 \%$ \\
\hline No Gloves oral medications & $100 \%$ & $100 \%$ \\
\hline
\end{tabular}

\subsection{Stage 3: Inter-rater agreement}

Graduate students enrolled in occupational safety and health programs, received training on the use of the tool, followed by assessment using video simulated home healthcare tasks for use as assessing inter-rater agreement and accuracy. The categorical data variables were either observed (1) or not observed (0) by the raters. The percent agreement and accuracy ranged from $50 \%-100 \%$, with an overall percent agreement and accuracy mean of $89.5 \%$. Results are presented in Table 2. The item that received $50 \%$ agreement was related to whether the cleaning product in the video was in a "manufacturer's container". A corresponding item, wipe disinfectant was noted by each of the raters ( $100 \%$ agreement). For the two items related to hand hygiene, the rater noted that hand hygiene was complete with soap and water and dried with a disposable towel; however, it was incorrectly classified as 
after patient care, not before patient care as demonstrated in the video. For three items in one video, the rater omitted any answer for badge present, last name, and working alone. In another video, one rater omitted observing cluttered walkway inside and out and a stair without railing. The raters' errors ranged from 1-6 items, with one rater having 1 error and another having 6 errors. Items with a percent agreement less than $100 \%$ were re-evaluated for clarity and correct answers were reviewed with the four raters.

Twenty-two items within the observation assessment tested the agreement of raters when observing frequency of position during worker tasks. For each item a mean and standard deviation were calculated. The standard deviation ranged from 0 to 8.62 , indicating there were some items with no variability $(\mathrm{SD}=0)$ between mean of the individual rater's frequency to very high variability $(\mathrm{SD}=8.62)$ in the difference between the mean of the frequencies documented as observed by the raters.

\section{Discussion}

Content development, content validity, and inter-rater agreement of the Home Healthcare Worker Observation Tool were the initial steps of this study aimed to assess and describe the occupational exposures HHCWs encounter within the home care environment.

\subsection{Conditions of work}

The developed content representing the workplace hazards within the home healthcare environment are consistent with "conditions of work" within the Conceptual Model for Integrated Approach for Worker Health and Safety. ${ }^{[16]}$ Elements of the physical environment, organization of work, and job tasks are captured within the observation tool as they have the potential to impact worker outcomes such as illness, injury, and wellbeing.

\subsection{Previous studies influence the HHCW observation design}

The tools designed for previous studies related to the health and safety of HHCWs were focused on workplace hazards, and like this tool, they had their focus and their limitations. The tool described by Polivka et al. ${ }^{[14]}$ was focused on home healthcare providers (nurses, aides, therapists) recall of occupational exposures within each room of a patient home with the goal to design training for future intervention studies. Interpersonal violence was specifically excluded from the study as needed to focus on the physical hazards. In contrast, the Home Healthcare Worker Observation Tool in the current study is designed to capture violence and factors that may lead up to violence or factors that can cause poor outcomes if

Published by Sciedu Press violence were to occur during a home care visit. Examples of factors that may lead to violence and are included within the tool are a patient experiencing confusion, $\mathrm{HHCW}$ working alone, un-restrained pets, and threats of violence.

In another study ${ }^{[11]}$ home care aides were asked to recall the hazards they encountered in the last five home care visits for some items and recall hazards encountered in the past 12 months for other items. These items were specifically inquiring about the existence of hazards or health outcomes such as low back pain. The number of respondents $(\mathrm{n}=$ 3,484 ) to the survey provided great insight into the hazards HHCWs encounter and provided foundational knowledge used to build into workplace assessments.

In another study, scripted interview questions allowed for focus groups and in-depth interviews of workers, union representatives, and home care patients to describe the occupational hazards encountered within home healthcare. ${ }^{[10]}$ The findings of this study identified hazards that impact the worker and the patient and the need for a household safety checklist and communication of hazards within the home. Similarly, the Home Healthcare Worker Observation Tool is designed with the interconnectedness of worker and patient safety in mind. Hand hygiene, environmental respiratory exposures, and violence all have the potential to impact the health and safety of the worker and the patient.

Gershon et al. ${ }^{[8]}$ designed and piloted a 96-item questionnaire, completed by 738 RNs working in home care. The items focused on physical hazards within the environment, violence and characteristics such as illicit drug use that could increase the risk of violence, and organizational characteristics that could perpetuate hazard exposure such as working alone and lack of safety equipment, and type of home and community in which the patient was located. This study informed development of the Home Healthcare Worker Observation Tool, which will allow researchers to identify if there are significant associations between the variables within the 12 sections of the tool.

Hittle et al. ${ }^{[2]}$ used a 69 -item scripted interview to assess the occupational hazards encountered by home care nurses and aides within the patients' home. The study provided insight that HHCWs have similar hazards based on providing care to a patient and different hazards based on the type of care provided. Hittle et al. ${ }^{[2]}$ indicated worker's risks were not limited by their scopes of practice as sometimes workers performed tasks outside their scope of practice. It is these details that informed the HHCW observation study to include any workers that enter the home, observing for tasks completed and hazards encountered. In addition to informed development of the observation tool, the researchers wanted 
to validate the content of the tool as it represented a wide range of hazards.

\subsection{Impact of content validity}

The S-CVI of .90 provides evidence that the observation tool provides a valid representation of the occupational hazards HHCWs encounter in the home healthcare environment. Reviewers' feedback allowed for refinement, removal, and addition of items that were not evident through a literature search alone. Through engagement of clinical and research experts in home healthcare and occupational health during development and content validity, items were verified to be relevant or not relevant to the concept of home healthcare hazards.

Items omitted during tool development were added to gain holistic insight into the breadth of occupational hazards in home care.

While the resulting instrument consist of 636 items, it is important to note that some sections and items included in the tool may not be used in each visit. Hittle et al. ${ }^{[2]}$ noted that when home health care nurses and home health care aides were interviewed, it was identified that depending on the type of work (e.g., nurse or aide) the worker performed different tasks and therefore was subjected to different hazards. The intention and design of the tool allows for many different types of HHCWs (nurse, therapist, aide, chaplain, social worker, medical provider) to be observed using the same tool. For example, if a nurse is observed and does not complete any cleaning tasks, 199 items will not be observed during that visit.

The tool is designed to be used as a checklist, providing the investigator with areas to quickly apply a checkmark to document an observed hazard (e.g., mold, patient confusion, cluttered walkway) and hashmarks to count the number of times a worker encounters other hazards (e.g., uncapped needle, ingress with equipment, bending to reposition a patient in bed). This design simplifies data collection and limits the investigator's need to write a narrative account of the observation. The tool is designed to capture potential risks to the worker and the patient.

\subsection{Effect of training}

Inter-rater agreement by four trained raters allowed the researcher to determine the training was effective, providing enough guidance for the raters to use the developed tool and assess the simulated home care environment with high percent agreement for a sample of categorical variables and low variability on items recorded as frequencies. Percent agreement overall was $89.5 \%$ and individual item scores provide the researchers guidance of which items will need to be retrained. The researchers desire $100 \%$ agreement to ensure the raters are capable of identifying hazards and provide $100 \%$ accuracy when identifying a hazard. The use of percent agreement was easily interpretable and provided clarity of which items the raters needed more training on. $\mathrm{McHugh}^{[24]}$ indicates when raters are well trained and minimal guessing is expected, percent agreement is a reliable measure.

In comparison to previous studies, items of which observers ergonomically assessed workers doing manual handling of patients, agreement among raters was fair to excellent. Karstad et al. ${ }^{[25]}$ found that agreement ranged from 55\%$100 \%$ when observing manual handling activities during eldercare and lower agreement was explained by smaller and more spontaneous body movements being difficult to distinguish during observations. The high variability on frequency item scores convey a need (1) for consideration of simplification of the tool to capture frequency items associated with ergonomics and (2) to develop multiple scenarios for raters to use in a live simulation with the trainer providing guidance and clarity on the use of the tool. Repeated use of the tool will ideally increase familiarity of the items on the tool and accuracy amongst raters when observing and documenting the occupational hazards.

\subsection{The importance of a workplace hazard assessment}

A workplace hazard assessment is one way to identify hazards and opportunities for healthier work practices to affect better outcomes for workers. ${ }^{[15]}$ The observation tool could serve as an assessment to investigate workplace hazards. In 2012, a panel of experts from industry, academia, and government agencies emphasized the connection between worker safety and patient safety and the need to recognize, understand, and measure safety concerns through hazard analysis. ${ }^{[26]}$ Identifying hazards is the first step to mitigating risks associated with hazards in the home care environment. The design of this tool allows for assessment of elements of healthcare that impact both worker and patient safety. For example, hand hygiene is assessed as completed and whether it was completed before or after patient care. Hand hygiene is a basic infection prevention tool that decreases transmission between workers and patients, potentially benefitting the worker, patient, and healthcare organization. ${ }^{[26]}$ The tool also assesses other aspects that potentially impact the worker, patient, and healthcare organization including: slip, trip, and fall hazards; safe patient handling; hazards related to the use of sharps; potential for violence within the home and community; and injury surveillance. ${ }^{[26]}$ The tool will allow the researcher to analyze areas of safety related to home care that has the potential to impact both worker and patient safety.

Previous studies have provided great insight into the per- 
ceived workplace hazards HHCWs encounter; however, they rely on worker and employer recall and do not provide the occupational health professional with firsthand assessment of the workplace. ${ }^{[2,11,12]}$ The tool is necessary to guide the occupational health professional in observing the hazards in the home care environment. To the researchers' knowledge, no tool currently exists to assess the range of potential hazards in the occupational environment of the home care setting. In order to understand and mitigate occupational hazards for this group of workers, we must have first-hand knowledge of their exposures during the tasks they complete in the home setting. After occupational health and safety professionals understand the hazards encountered and the association of organization of work within the home, researchers can move forward to mitigate hazards in an effort to better protect HHCWs through informed safety training, intervention studies, and changes to practice and policy.

\subsection{Other considerations}

One limitation within this study was related to the interrater percent agreement, in that, percent agreement does not consider that agreement by chance and included a limited number of raters. These raters were trained with consistent results related to identifying hazards. The inter- rater agreement assessment and outcome analysis is specific to the four participating raters and these results are not generalizable to other raters. During planned pilot testing of the tool, additional psychometric testing needs to include reliability with additional raters, internal consistency and factor analysis.

Items within the observation tool are limited to those items that are observed within the space the worker is working, dur- ing a specific visit, and related to the tasks being performed. For example, if the worker does not enter the basement, the researcher does not capture the mold and pesticides that the worker may encounter on other visits when they enter the basement to do laundry.

\section{RELEVANCE TO NURSING RESEARCH}

Following a trusted process for tool development, establishing content validity, and analyzing initial inter-rater agreement were essential first steps to designing a tool that is both reliable and valid to define the occupational hazards in the home healthcare environment. Content validity provided support for most items within the tool and insight as to what items were omitted and needed to be added. The developed online training and assessment for the raters proved to be adequate as a training method.

Workplace hazard assessments are needed to understand the occupational hazards that HHCWs encounter. The need to determine the hazards for this healthcare group is specifically crucial given the rapid expansion of the home healthcare industry. Studies that use trained observers in the home care environment to assess the work tasks and environmental factors have the potential to affect the health and safety of the growing population of HHCWs. This validated observation tool will provide a data collection instrument for researchers investigating the breadth and frequency of hazards HHCWs encounter.

\section{CONFLICTS OF INTEREST Disclosure}

The authors declare that there is no conflict of interest.

\section{REFERENCES}

[1] U.S. Bureau of Labor Statistics. 2020. Employment projection. Available from: https://data.bls.gov/projections/nationalM atrix?queryParams $=621600 \&$ ioType $=i$

[2] Hittle B, Agbonifo N, Suarez R, et al. Complexity of occupational exposures for home health-care workers: Nurses vs. home health aides. Journal of Nursing Management. 2016; 24: 1071-1079. PMid:27406330 https://doi.org/10.1111/jonm. 12408

[3] National Institute for Occupational Safety and Health. Occupational health supplement: National Health Interview Survey. Figure 4: Prevalence of current asthma among U.S. adults who worked in the past 12 months. 2010. Available from: https://www. cdc.go v/niosh/topics/nhis/healthcareind/hcindfig4.html

[4] U. S. Bureau of Labor Statistics, U. S. Department of Labor. Injuries, illness, and fatalities: Table 1. Incidence rates of nonfatal occupational injuries and illnesses by industry and case types, 2018. 2019. Available from: https://www.bls.gov/web/osh/summ1_00.h tm

Published by Sciedu Press
[5] Davis KG, Kotowski SE. Prevalence of Musculoskeletal Disorders for Nurses in Hospitals, Long-Term Care Facilities, and Home Health Care. Human Factors: The Journal of the Human Factors and Ergonomics Society. 2015; 57(5): 754-792. PMid:25899249 https://doi.org/10.1177/0018720815581933

[6] Gershon R, Pearson J, Sherman M, et al. The prevalence and risk factors for percutaneous injuries in registered nurses in the home healthcare sector. American Journal of Infection Control. 2009; 37: 525533. PMid:19216006 https://doi.org/10.1016/j.ajic. 200 8.10 .022

[7] Quinn MM, Markkanen PK, Galligan KD, et al. Sharps injuries and other body fluid exposures among home health care nurses and aides. American Journal of Public Health. 2009; 99(S3): S710-717. PMid:19890177 https://doi.org/10.2105/AJPH. 2008.1501 69

[8] Gershon RRM, Canton AN, Raveis VH, et al. Household-related hazardous conditions with implications for patient safety in the home health care sector. Journal of Patient Safety. 2008a; 4(4): 227-234. https://doi.org/10.1097/PTS.0b013e31818936e3 
[9] Gershon RRM, Porgorzelska M, Qureshi KA, et al. Home health care patients and safety hazards in the home: Preliminary Findings. In Henricksen, K., Battles J. B., Keyes, M. A. et al., (Eds.) Advances in Patient Safety: New Directions and Alternative Approaches. (Volume 1: Assessment). Agency for Healthcare Research and Quality. 2008b.

[10] Markkanen P, Quinn M, Galligan C, et al. Characterizing the nature of home care work and occupational hazards: A developmental intervention study. American Journal of Industrial Medicine. 2014 57(4): 445-457. PMid:24347541 https://doi.org/10.1002/aj im. 22287

[11] Quinn MM, Markkanen PK, Galligan CJ, et al. Occupational health of home care aides: Results of the safe home care survey. Occupational \& Environmental Medicine. 2016; 73(4): 237-245. PMid:26209318 https://doi.org/10.1136/oemed-2015-103031

[12] Agbonifo N, Hittle B, Suarez R, et al. Occupational exposures of home healthcare workers. Home Healthcare Now. 2017; 35(3): 150159. PMid:28248782 https://doi.org/10.1097/NHH.000000 0000000509

[13] Markkanen P, Galligan C, Quinn M. Safety risks among home infusion nurses and other home health care providers. Journal of Infusion Nursing. 2017; 40(4): 215-223. PMid:28683000 https: //doi.org/10.1097/nan.0000000000000227

[14] Polivka BJ, Wills CE, Darragh A, et al. Environmental health and safety hazards experienced by home health care providers: A roomby-room analysis. Workplace Health \& Safety. 2015; 63(11): 512 522. https://doi.org/10.1177/2165079915610532

[15] Centers for Disease Control and Prevention (2019). Workplace health promotion: Environmental assessment. Available from: https://www.cdc.gov/workplacehealthpromotion/m odel/assessment/environmental.html

[16] Sorensen G, McLellan DL, Sabbath EL, et al. Integrating worksite health protection and health promotion: A conceptual model for intervention and research. Prevention Medicine. 2016; 91: 188-196. PMid:27527576 https : //doi .org/10.1016/j.ypmed. 2016.0 8.005
[17] Beck CT, Gable RK. Ensuring content validity: An illustration of the process. Journal of Nursing Measurement. 2001; 9: 201215. PMid:11696942 https://doi.org/10.1891/1061-3749. 9.2. 201

[18] Lynn MR. Determination and quantification of content validity. Nursing Research. 1986; 35: 382-385. https : //doi .org/10.1097/00 006199-198611000-00017

[19] Wittemore R, Knafl K. The integrative review: Updated methodology. Journal of Advanced Nursing. 2005; 52: 546-553. PMid:16268861 https : //doi.org/10.1111/j.1365-2648.2005.03621.x

[20] Polit DF, Beck CT. The content validity index: Are you sure you know what's being reported? Critique and recommendations. Research in Nursing and Health. 2006; 29: 489-497. PMid:16977646 https://doi.org/10.1002/nur. 20147

[21] Gisev N, Bell JS, Chen TF. Interrater agreement and interrater reliability: Key concepts, approaches, and applications. Research in Social and Administrative Pharmacy. 2013; 9: 330-338. PMid:22695215 https://doi.org/10.1016/j.sapharm.2012.04.004

[22] Polit DF, Beck CT. Nursing research: Generating and assessing evidence for nursing practice. 10th ed. Wolters Kluwer/Lippincott Williams \& Wilkins. 2017.

[23] Barrows HS. Simulated (standardized) patients and other human simulations. Health Sciences Consortium. 1987.

[24] McHugh ML. Interrater reliability: The Kappa statistic. Biochemia Medica. 2012; 276-282. PMid:23092060 https ://doi.org/10.1 $1613 / \mathrm{bm} .2012 .031$

[25] Karstad K, Rugulies R, Skotte J, et al. Inter-rater reliability of direct observations of the physical and psychosocial working conditions in eldercare: An evaluation in the DOSES project. Applied Ergonomics. 2018; 69: 93-103. PMid:29477334 https ://doi.org/10.1016/ j.apergo.2018.01.004

[26] Joint Commission. Improving patient and worker safety: Opportunities for synergy, collaboration, and innovation. 2012. Available from: https : //www. jointcommission.org/assets/1/18/TJ C-ImprovingPatientAndWorkerSafety-Monograph.pdf 